\title{
SELECTED ABSTRACTS OF PAPERS FROM OTHER JOURNALS
}

Spinal cord damage in surgery of the abdominal aorta, by D. E. Szilagyi, J. H. Hageman, R. F. Smith and J. P. Elliott. Surgery, 83: 38-56, January 1978.

The incidence, aetiological and clinical features of myelopathy secondary to abdominal aortic surgery and aortography are reviewed on ground of the authors' experience in ten cases and of a survey of 36 cases reported in the literature. From 1952 to 1977 the authors report a personal incidence of 0.25 per cent (eight of 3.164 ) and of 0.01 per cent (two of 17.949 ) cord damage following aortic operations and abdominal aortograms, respectively. Postoperative spinal cord damage was ten times more frequent in ruptured than in unruptured abdominal aneurysms (five of 247 cases $v s$. three of $\mathrm{I} \cdot 477$ cases). The neurological deficit in eight patients was a flaccid complete paraplegia in five (62 per cent) and an incomplete one in two ( 25 per cent) with a high rate of deaths, three of eight (38 per cent).

The factor which causes postoperative cord damage is ischaemia. It depends on the anatomical disposition of the great radicular artery (GRA) on the possible substitute of infrarenal lumbar radicular arteries, and on the richness of the potential collateral circulation. High aortic clamping which may interfere with the GRA and hypotension reducing perfusion capabilities increased the probability of cord ischaemia. In postaortographic cases damage to the cord is more infrequent and shows a wider scale of severity. It is caused by a chemical insult which results from direct introduction of contrast material into the spinal cord blood supply with some hypersensitivity reaction of the cord substance.

This is an excellent review article on this topic.

Spectrum of the hangman's fracture, by E. L. Seljeskog and S. N. Chou. Fournal of Neurosurgery, 45: 308, July 1976.

The authors review their experience in managing 26 cases of 'hangman's fracture'. The basic mechanism of injury was hyperextension of the upper cervical spine. Radiographic studies revealed a spectrum of injury beginning with the classical hangman's fracture and progressing to the simple $\mathrm{C}_{2}$ laminar-pedicle fracture. Appreciation of the fracture instability will dictate the method and duration of fracture treatment. Management by a closed, nonoperative means resulted in solid union of the bone in all fully treated cases, with a minimum of morbidity.

Anterior approach to the thoracolumbar spine, by J. D. Burrington, C. Brown, R. Wayne and J. Odom. Arch. Surg., III: 456-463, April 1976.

Forty-five patients, of whom most were children, underwent extensive exposure of the thoracolumbar spine to correct serious orthopaedic abnormalities. The spine was exposed through a combined thoracotomy and retroperitoneal approach that gave excellent access with minimal morbidity. The diaphragm was opened circumferentially after the peritoneum had been dissected from its muscular portion. This permitted repair of the diaphragm with no detectable loss of function. Although this approach was developed for exposure of the spine, it can also be utilised to expose the entire aorta, both kidneys and their blood supply, and the retroperitoneal area for possible excision of large tumours.

Discontinuity of the spinous process on standard roentgenographs as an aid in the diagnosis of unstable fractures of the spine, by T. Chiroff and B. L. Sachs. fournal of Trauma, 16: 313-316, April 1976.

It has been observed that discontinuity in the outline of the spinous process on standard anteroposterior radiographs is a characteristic finding in some unstable spine fractures. 
Since the spinous processes are often not depicted clearly on lateral views of the trauma victim's spine, these are less reliable; however, if a transverse fracture through the spinous process at the level of, or adjacent to, an anterior compression fracture is demonstrable, this confirms the unstable nature of the injury. Recognition of this sign, when present, may eliminate excessive pretreatment manipulations in some patients who have sustained major trauma and are suspected of having unstable spine fractures.

Postural hypoxemia in quadriplegic patients, by A. L. Goldman and J. George. Neurology, 26: 81 5-8I7, September I976.

Quadriplegic patients frequently undergo unusual positional changes, including headdown tilt. To determine if hypoxaemia develops in this position and whether it is related to the duration of quadriplegia, five patients were studied within I year of injury (group A) and five after I year from injury (group B). Arterial blood gases were obtained in the seated and 45-degree head-down position. Group A patients had a decrease in arterial partial pressure of oxygen in the head-down position $(p<0.025)$, while group B patients did not $(p>0.4)$. This study demonstrated that quadriplegic patients have positional hypoxaemia early in their course. This probably results from relative hypoventilation of the upper lung fields caused by flaccid paralysis of the intercostal muscles. The positional hypoxaemia disappears with time, presumably because of the change from flaccid to spastic paralysis of the intercostal muscles.

Possible mechanisms for observed pathophysiological variability in experimental spinal cord injury by the method of Allen, by S. H. Koozekanani, W. M. Vise, R. M. Hashemi and R. B. McGhee. F. Neurosurg., 44: 429-434, April 1976.

Experimental spinal cord injuries were induced in dogs by dropping calibrated weights through a vented tube onto a small impounder resting on the surgically exposed cord. The motion of the impounder and the drop-mass were recorded by high-speed photography and the resulting data were compared to those obtained from a computer simulation of the dynamics of the injury mechanism. It is concluded that this method of inducing spinal cord injuries may yield markedly different degrees of cord compression depending upon the parameters of the animal material and apparatus even when the gm-cm of impact energy is maintained at a constant value. Some approaches to standardisation of this injury model are suggested.

Sweating responses to central and peripheral heating in spinal man, by J. A. Downey, C. E. Huckaba, P. S. Kelley, H. S. Tam, R. C. Darling and H. Y. Cheh. Fournal of Applied Physiology, 40: 701-706, May 1976.

Studies of central and peripheral heating of a resting spinal man (T6)were performed under various ambient temperatures $\left(20-34^{\circ} \mathrm{C}\right)$. It was found that at a constant core temperature, sweating could not be initiated by sentient skin heating alone, but skin cooling alone did produce a rapid decrease in sweating response. Central heating alone induced sweating responses and the central temperature thresholds of sweating were inversely related to the ambient (sentient skin) temperatures. The local and mean sweating rates were found to be linearly related to the core temperature. The slopes of local sweating rates versus the core temperature vary increasingly with the following locations: chest, forearm, and forehead; but the slopes of mean sweating rates versus core temperature were essentially constant.

Die Hand des Tetraplegikers, by G. Zrubecky. Unfallheilkunde, 79: 45-54, February 1976.

The restoration of prehensile function in high tetraplegics should be the central aim of treatment. The functional-anatomical motor loss of functions at the various levels of 
injury is outlined and in particular the muscle groups with residual functions and the compensatory movements thereby are discussed. From this the possible conservative and surgical consequences are derived. The possibilities of providing a paralysed hand with a myoelectric or reciprocal prothesis are of utmost significance in the conservative stage of treatment. The technical construction of both kinds of prothesis are described. Additionally, the paper deals with the indications for surgical reconstruction of prehensile function. Performance and results of the operation, which was developed in Tobelbad for a C6 lesion, are outlined.

The effects of some anticholinergic compounds on the infused urinary bladder of anaesthetized rabbits, by C. Sjögren. Acta Pharmacol. et Toxicol., 39: 167-176.

The intravesical pressure and volume of the urinary bladder of anaesthetised rabbits were studied using a urinary bladder infusion technique. It was shown that the anticholinergic compounds used, atropine, emeprone, and PR I97, significantly increased the urinary bladder volume capacity, and that they decreased the micturition pressure. Atropine was the most potent compound in increasing the bladder volume capacity, followed by PR I 97 and emeprone, mentioned in order of potency. Atropine and PR I97 increased the bladder volume capacity and the micturition threshold pressure significantly more than emeprone. There was no statistically significant difference between the effects of the different compounds on the residual volume or the micturition pressure. It is suggested that the effects of the compounds are confined mainly to the bladder and most probably to its cholinergic structures.

The effect of antihistamines on experimental post-traumatic edema of the spinal cord, by A. I. Kobrine, T. F. Doyle and H. V. Rizzoli. Surgical Neurology, 5: 307-309, May 1976.

In this experiment we studied the effect of antihistamines on post-traumatic experimental spinal cord oedema. One group of five animals was treated with chlorpheniramine and metiamide, potent antihistamines which together block both the $\mathrm{HI}$ and $\mathrm{H} 2$ receptor sites. A second group of five animals was untreated and served as controls. All animals received a $600 \mathrm{gm} \mathrm{cm}$ injury to the dural covered spinal cord at Tro. Oedema formation was measured at six hours post injury, using the RISA technique. No significant difference was found in the amount of oedema in the treated and untreated groups. We conclude from this data that the breakdown in the blood brain barrier and resultant oedema formation which accompanies experimental traumatic spinal cord injury is not a histamine mediated phenomenon.

\section{Norepinephrine levels in traumatized spinal cord of catecholamine-depleted cats, by T. W. Schoultz, D. C. DeLuca, and D. L. Reding. Brain Research, I09: 367-374, 1976 .}

This study was designed to elucidate the origin of norepinephrine (NE) measured in spinal cord following trauma. In normal cats the NE concentration at the lesion site increased 63 per cent over control I hour following experimentally produced blunt trauma $(4005-\mathrm{cm})$. Spinal cords of adrenalectomised cats were also traumatised, but there was no increase in NE levels I hour post-trauma. Spinal cord NE levels in these animals were not significantly different from normal or adrenalectomised non-injured controls. In the absence of one of the major peripheral sources of catecholamine, the adrenal medulla, our decreased NE levels prompt us to disagree with the hypothesis that $\mathrm{NE}$ measured in injured spinal cord is liberated from intrinsic neuronal systems. It is likely that the mechanism of $\mathrm{NE}$ accumulation is directly related to increased circulating levels of NE. The authors speculate on a possible reason for the conflicting results obtained by different laboratories in this area of research. 
Sympathetic activity in the proximal urethra in patients with urinary obstruction, by S. A. Awad, J. W. Downie, D. W. Lywood, R. A. Young and S. V. Jarzylo. fournal of Urology, I15: 545-547, May 1976.

A I5-year experience with I6 patients who suffered complete rupture of the membranous urethra and obliteration of the apex of the dislocated prostate gland is described. In two cases repair was done immediately after the injury. The technique is described. Entrance into the perineum follows the path of the bulbous urethra rather than that of the rectal wall. After the bulbous urethra is mobilised the urogenital diaphragm is excised. The end of the bulbous urethra is approximated to the mobilised distal half of the prostate, via the shortest route between the two organs.

Penile prosthesis: psychologic factors, by T. D. Stewart and S. N. Gerson. Urology, 7: 400-402, April 1976.

A 3 I-year-old paraplegic male requested a penile prosthesis. Psychiatric evaluations were done of both the patient and his wife. Within 72 hours of his arriving home with the prosthesis installed, his wife left their house to seek a divorce. The implications of this event for selecting patients of such devices are explored. Reservations are expressed about a prosthesis being inserted for patients married after the onset of impotence.

Intraurethral pressure recording by $M$. Asmussen. Scandinavian fournal of Urology and Nephrology, 10: I-6, 1976.

This paper presents the results of a comparative study of intraurethral pressure recording with two different catheter systems: (I) Open-end water-filled catheters with constant flow connected to conventional pressure transducers, and (2) Microtransducers mounted in the tip of a dacron catheter. When open-end catheters were used, the best reproducibility of the urethra pressure profile was obtained, if the catheter dimensions $6-8 \mathrm{~F}$ were used and if the flow in the catheters was kept to approximately $\mathrm{I}-2 \mathrm{ml} / \mathrm{min}$. In addition, the withdrawal rate of the open-end catheters should not exceed $3-4 \mathrm{~mm} / \mathrm{sec}$. The best reproducibility of the intraurethral pressure was obtained with the microtransducer. It was free from measuring artefacts and seemed from this investigation superior to the open-end catheter systems, both for clinical practice and for research.

Urodynamic evaluation of a chronically implanted bladder pacemaker, by L. W. Jones, U. Jonas, E. A. Tanagho and J. P. Heine. Investigative Urology, 13: 375-379, 1976.

A 'chronic' study of an implantable bladder pacemaker revealed that, although the electrode array did not significantly impair vesical function during the Io weeks of research, electric stimulation did spread to the voluntary perineal musculature. There was also a drop in bladder response to the same stimulation parameters, probably due to progressive development of fibrous reaction and encasement of electrodes.

Sexual adjustment and feminine attractiveness among spinal cord injured women, by S. Bregman and R. G. Hadley. Archives of Physical Medicine and Rehabilitation, 57: 448-450, September 1976.

Responses to semistructured interviews with 3I spinal cord injured women yielded sexual adjustment scores and material concerning sexual compensation and orgasm, bowel and bladder programmes, and methods of enhancing attractiveness. Sexual adjustment scores were unrelated to time since injury. Few subjects reported the need to make special provision to control elimination during sexual activity. Openness and honesty with mates were stressed. Those with high sexual-adjustment scores compensated for lost sensation and mobility. Orgasm descriptions and most attractivenessrelated behaviours resembled those of able-bodied women; some attractiveness behaviours specifically compensated for disability. 